\title{
Uso de conjuntos difusos para cuantificar el potencial de cuerpos académicos desde la conformación de redes científicas de colaboración
}

\author{
Fuzzy sets implementation to quantify the potential for academic \\ groups from the formation of scientific collaboration networks
}

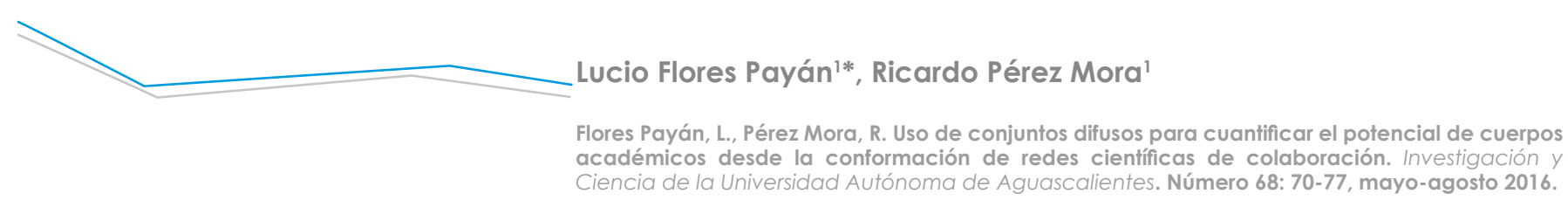

\section{RESUMEN}

El establecimiento de redes de colaboración científicas de Cuerpos Académicos, se presenta al paso del tiempo como un elemento estratégico para la generación y difusión de conocimiento. Es en este sentido que el análisis y estudio de estas redes y sus resultados se presentan de forma pertinente; sin embargo, es un fenómeno complejo y multidimensional. Es por esto que se requieren nuevas formas y herramientas para su estudio. El presente trabajo indaga en la teoría de la lógica difusa como elemento de análisis que permite de forma precisa una distinción en la cuantificación del potencial de los Cuerpos Académicos de forma multidimensional; se hace evidente el mayor potencial que tienen los cuerpos académicos que conforman redes de colaboración científica.

\section{ABSTRACT}

The establishment of networks of scientific collaboration of academic groups occurs like a strategic element for the generation and dissemination of knowledge. It is in this sense that the analysis and study of these networks and their results are presented in an appropriate

Palabras clave: grupos académicos, redes de colaboración, lógica difusa, conjuntos difusos, redes institucionales, producción de conocimiento.

Keywords: academic groups, networks, fuzzy logic, fuzzy sets, institutional networks, knowledge generation.

Recibido: 11 de febrero de 2015, aceptado: 28 de enero de 2016

Departamento de Políticas Públicas, Universidad de Guadalajara.

* Autor para correspondencia: florespayan@hotmail.com way, however, it is a complex and multidimensional phenomenon. This is why new methods and tools are required. This paper explores the theory of fuzzy logic as an element of analysis that allows to accurately distinguish in quantifying the potential of academic groups in a multidimensional way. It makes evident the greater potential of academic bodies that make scientific collaboration networks.

\section{INTRODUCCIÓN}

El comportamiento de las sociedades actuales como un fenómeno social hace evidentes nuevas necesidades, que en mayor medida son canalizadas en elementos científico-tecnológicos, una reorganización de la base sociocultural anticipada por el reacomodo del mercado y su dependiente economía, todo esto demanda formas más integrales de generación de conocimiento científico y su pertinente difusión y propagación, de tal manera que faculte el entendimiento de estos comportamientos contemporáneos para la compresión de este fenómeno social.

En ese sentido, la conformación de redes juega un rol central como estrategia consensada por los integrantes para potencializar la generación de conocimiento, transmisión de información y difusión de la influencia de algunas organizaciones en el ámbito social (Kossinets y Watts, 2009). El concepto de redes es utilizado en diferentes esferas y ámbitos, algunas principales son: social, político, tecnológico, industrial y en mayor medida cibernético.

La producción de conocimiento científico y tecnológico ha cobrado un papel fundamental en el mercado internacional y es señalado como uno de los 
factores clave para la integración y competitividad dentro del contexto económico global (Banco Mundial, 2003). Es así que la conformación de redes en general y de las redes de colaboración académicas en particular cobra un alto valor, porque permite a los académicos y científicos trabajar con flexibilidad e integración.

El presente trabajo establece como hipótesis central la importancia que encuentra la integración de redes de colaboración en la esfera científica y académica, esto en términos de la generación de conocimiento, su difusión y divulgación, así como para la transmisión de información. Lo anterior bajo el supuesto de que a partir del intercambio de ideas como trabajo colaborativo se logra potencializar la generación de conocimiento.

Los llamados cuerpos académicos (CA) como una forma o modo colectivo de producir conocimiento pueden verse constituidos como tejidos académicos de trabajo, y de esta forma ser estudiados bajo los criterios de las redes para generación de conocimiento. En este sentido, el presente trabajo tiene como objetivo elemental cuantificar el potencial que podrían alcanzar los cuerpos académicos promovidos por el Programa para el desarrollo profesional docente (PRODEP) en México, a partir de dos ejes analíticos: a) la construcción de redes y b) los elementos de investigación. Para alcanzar este objetivo, se desarrolló un Índice difuso del potencial de los cuerpos académicos (IDPca), que fue construido a partir de los elementos de la teoría de la lógica difusa, esto con la intención de determinar de forma más puntual y multidimensional los elementos mediante los cuales puede ser cuantificado el nivel de desarrollo que podrían alcanzar los CA a partir de la construcción de redes de colaboración.

\section{MATERIALES Y MÉTODOS}

Jackson y Wolinsky (1996) exponen un procedimiento para la cuantificación de la utilidad de una red en términos de conectividad entre sus integrantes, para fines del presente trabajo se ha retomado esta propuesta adaptándola en términos de cuantificar la utilidad a partir de la producción y desarrollo de actividades de los CA, esto con la intención de hacer visible un comparativo entre el potencial de desarrollo que logran los CA que establecen redes de colaboración contra los que no hacen este tipo de redes.
En la revisión de literatura relacionada con el tema de redes, Salas y Murillo (2014) proponen una serie de supuestos para el análisis, que para fines del presente trabajo son retomados como elementos principales en los cuales se sustenta la construcción analítica y metodológica:

- El proceso considera la unión de más de dos agentes que desean formar, mantener 0 romper vínculos entre ellos (Jackson, 2005).

- Es necesario consentimiento mutuo, aunque la ruptura es unilateral pudiendo realizarse formal o informalmente (Carayol et al., 2008).

- Los agentes basan sus decisiones tanto en la utilidad inmediata que les generan los vínculos (Jackson, 2008; Carayol y Roux, 2009).

- Las redes son dinámicas y, por tanto, propensas a evolucionar (Jackson, 2005).

En el actual trabajo se utilizaron los datos generados a partir de la aplicación del cuestionario "Modos colectivos de producción de conocimiento en universidades Públicas Estatales" (UPE), mismo que se encuentra constituido por 628 observaciones con relación a las actividades y estado actual de los cuerpos académicos de 59 universidades de México -que son mostradas en los anexos de este trabajo-.

Seanalizaelpotencial delos cuerposacadémicos desde del trabajo colaborativo y la conformación de redes a partir de dos ejes analíticos: Investigación y Gestión. Se pretende proponer un modelo analítico que faculte la medición cuantitativa del potencial de cada CA, la idea tiene fundamento en la proposición de una función de utilidad que integre los factores que influyan en el potencial de un CA:

Potencial del $C A=f\left(\right.$ investigació $_{i}^{n}$, gestiones $\left._{i}^{n}\right)$

La anterior función de utilidad es desarrollada con conjuntos difusos, para lo cual fueron contemplados seis indicadores que representan actividades de investigación y gestión desempeñadas por los CA y se muestran en la Tabla 1.

Los conjuntos difusos han sido utilizados de manera más puntual para encontrar soluciones en procesos de ingeniería, debido a la factibilidad de realizar el mesurado de información subjetiva e imprecisa a partir de la creación de un sistema de 
Tabla 1. Indicadores utilizados para el cálculo del IDPca

\begin{tabular}{|c|c|c|c|}
\hline $\begin{array}{l}\text { Ejes de } \\
\text { análisis }\end{array}$ & Indicadores & Ejes de análisis & Indicadores \\
\hline \multirow{3}{*}{$\begin{array}{l}\text { Eje } 1 . \\
\text { Títulos } \\
\text { académicos }\end{array}$} & 1. Reconocimiento PRODEP & \multirow{5}{*}{$\begin{array}{l}\text { Eje } 4 . \\
\text { Actividades de } \\
\text { Gestión }\end{array}$} & \multirow{5}{*}{$\begin{array}{l}\text { 6. Actividades de vinculación } \\
\text { - Vinculación con } \\
\text { el sector social } \\
\text { (organizaciones } \\
\text { sociales, ONG) } \\
\text { - Vinculación con } \\
\text { el sector privado } \\
\text { (empresa, industria) } \\
\text { - Vinculación con } \\
\text { el sector público } \\
\text { (gubernamentales). }\end{array}$} \\
\hline & $\begin{array}{l}\text { 2. Reconocimiento del Sistema Nacional de } \\
\text { Investigadores (SNI) }\end{array}$ & & \\
\hline & 3. Título de Doctorado & & \\
\hline $\begin{array}{l}\text { Eje } 2 \text {. } \\
\text { Formación } \\
\text { de recursos } \\
\text { humanos }\end{array}$ & 4. Estudiantes en apoyo en el CA & & \\
\hline \multirow[t]{2}{*}{$\begin{array}{l}\text { Eje } 3 . \\
\text { Actividades } \\
\text { de } \\
\text { investigación }\end{array}$} & $\begin{array}{ll}\text { 5. } & \text { Tipo de actividades realizadas } \\
- & \text { Artículos en revistas indexadas } \\
\text { - } & \text { Artículos en revistas arbitradas } \\
\text { - } & \text { Capítulos de libro } \\
\text { - } & \text { Proyectos de investigación } \\
\text { - } & \text { Formación de grupos de investigadores } \\
\text { - } & \text { Patentes } \\
\text { - } & \text { Asetotipos } \\
\text { - } & \text { Consulas } \\
\text { - } & \text { Memorias en extenso } \\
& \text { Dirección de tesis }\end{array}$ & & \\
\hline & & $\begin{array}{l}\text { Eje } 5 \text {. } \\
\text { Intercambios } \\
\text { académicos }\end{array}$ & $\begin{array}{l}\text { 7. Intercambios } \\
\text { - Al extranjero } \\
\text { - Al interior del país } \\
\text { - En la entidad }\end{array}$ \\
\hline
\end{tabular}

Elaboración propia.

inferencia difuso (SID), el cual es conformado por un algoritmo de procesamiento de datos inexactos.

La relevancia de la creación de un SID es la posibilidad de la correspondencia no lineal entre una o varias variables de entrada y una variable de salida, pasando por un proceso de transformación y recuperación de los datos (fuzzificacióndesfuzzificación) (ver Figura 1).

\begin{tabular}{|c|c|c|}
\hline $\begin{array}{l}\text { Proceso de incorporación } \\
\text { de información (procesos } \\
\text { de fuzzificación). }\end{array}$ & $\begin{array}{l}\text { Proceso de operaciones } \\
\text { recursivas (proceso de } \\
\text { operaciones y reglas } \\
\text { difusas). }\end{array}$ & $\begin{array}{l}\text { Proceso de respuesta o salida } \\
\text { de información procesado } \\
\text { al medio (proceso de } \\
\text { desfuzzificación). }\end{array}$ \\
\hline $\begin{array}{l}\text { A través de la lógica difusa, } \\
\text { los modelos de lógica difusa } \\
\text { son capaces de introducir } \\
\text { a su dominio de operación, } \\
\text { una gran cantidad de } \\
\text { datos de diversa naturaleza. } \\
\text { Este proceso implica una } \\
\text { transformación de los datos } \\
\text { pertenecientes a un dominio } \\
\text { determinado a términos de } \\
\text { enunciados en un dominio } \\
\text { difuso. }\end{array}$ & $\begin{array}{l}\text { Al interior del sistema de } \\
\text { inferencia difusa, los datos } \\
\text { se encuentran ya en } \\
\text { dominio numérico y se tratan } \\
\text { como información para } \\
\text { ser procesada mediante } \\
\text { operaciones de integración, } \\
\text { comparación, ponderación y } \\
\text { con ello formar las funciones } \\
\text { de pertenencia de cada } \\
\text { valor. }\end{array}$ & $\begin{array}{l}\text { En la salida el sistema de } \\
\text { inferencia difusa, los valores } \\
\text { resultantes de las operaciones } \\
\text { de los conjuntos son } \\
\text { nuevamente trasladados a } \\
\text { términos propios del lenguaje } \\
\text { del entorno de análisis. }\end{array}$ \\
\hline
\end{tabular}

Figura1. Ełapas del sistema de inferencia difuso. Fuente: Flores y Camarena (2013). 
Zadeh (1965) fue uno de los pioneros en el diseño y uso de la estructuración de conjuntos difusos, concibiéndolos como una colección de objetos, donde a cada uno de estos objetos le corresponde una función de membresía, la cual asigna un cierto grado de pertenencia a un conjunto definido. La función de pertenencia distingue un rango que puede ser un conjunto de números reales no negativos, convencionalmente formada entre 0 y 1 y de forma continua como se muestra en la Figura 2.

$$
\mathrm{X} \mu_{\mathrm{A}} \rightarrow[1,0]
$$

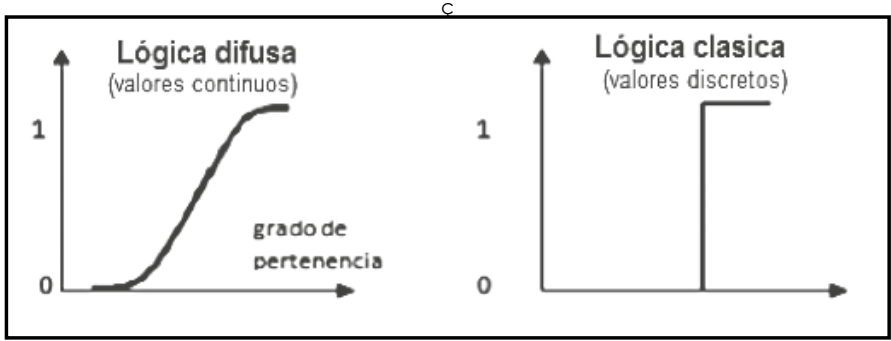

Figura 2. Lógica difusa vs. lógica clásica. Elaboración propia.

La representación matemática de un conjunto difuso cuando $X$ es una colección de objetos denotados por $\mathrm{X}, \mathrm{X}=\left\{\mathrm{X}_{1}, \mathrm{X}_{2}, \mathrm{X}_{3} \ldots\right\}$, así un subconjunto difuso $A$ en $X$ es un conjunto de pares ordenados:

$$
A=\{x, \mu \mathrm{A}(x), \mid x \in U\}
$$

En la investigación social la implementación de los conjuntos difusos ha sido realizada por autores como López et al. (2009), quienes realizan un modelo difuso para la descomposición y cuantificación multidimensional de la pobreza, asimismo, Flores Payán y García Batíz (2013) realizan la evaluación del programa público HÁBITAT, proponiendo un modelo basado en conjuntos difusos para la cuantificación del impacto que dicho programa alcanza en su población objetivo. De acuerdo con lo anterior, para el presente trabajo fueron construidos cinco sistemas de inferencia difusos (SID) para la cuantificación del potencial y la generación del IDPca, dichos SID se conformaron por los conjuntos referentes a cada uno de los ejes de análisis y sus particulares reglas difusas diseñadas a partir de tablas de verdad.

Para cada variable se construyó un conjunto difuso que hace uso de etiquetas lingüísticas para reflejar en un continuo el grado de potencial que puede alcanzar cada CA. Para fines explicativos, a continuación se detalla la construcción del conjunto asociado al indicador de porcentaje de miembros pertenecientes al Sistema Nacional de Investigadores (SNI), porcentaje de miembros que cuentan con reconocimiento PRODEP y porcentaje de miembros que cuentan con título doctoral de cada cuerpo académico.

La Figura 3 representa el conjunto difuso generado para la cuantificación del porcentaje de miembros pertenecientes al SNI de cada cuerpo académico y que está conformado por tres funciones de pertenencia, la etiqueta "malo" agrupa el más bajo porcentaje de miembros del $\mathrm{SNI}$ con relación al total de CA e igualmente para las otras dos funciones de pertenencia etiquetadas como "regular" y "buena".

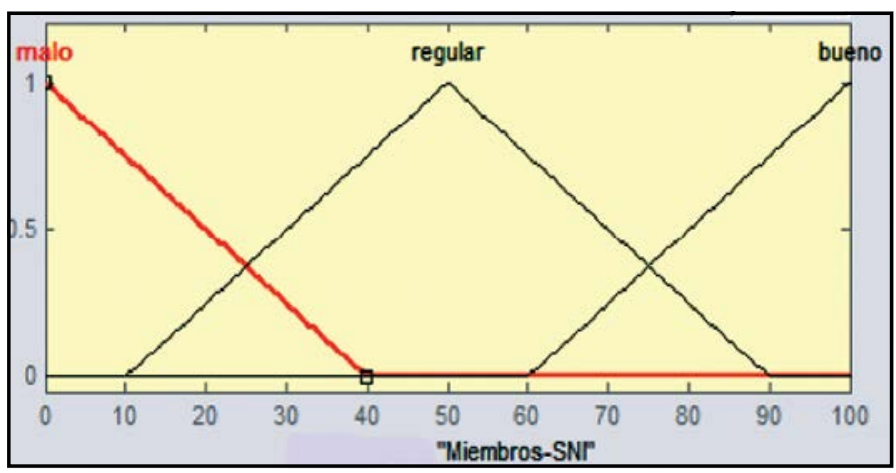

Figura 3. Conjunto difuso para el porcentaje de miembros en el SNI de los CA. Elaboración propia.

La integración de este tipo de conjuntos difusos perteneciente a cada indicador conformó el SID que se muestra en la Figura 4 y que representa el eje 1 .

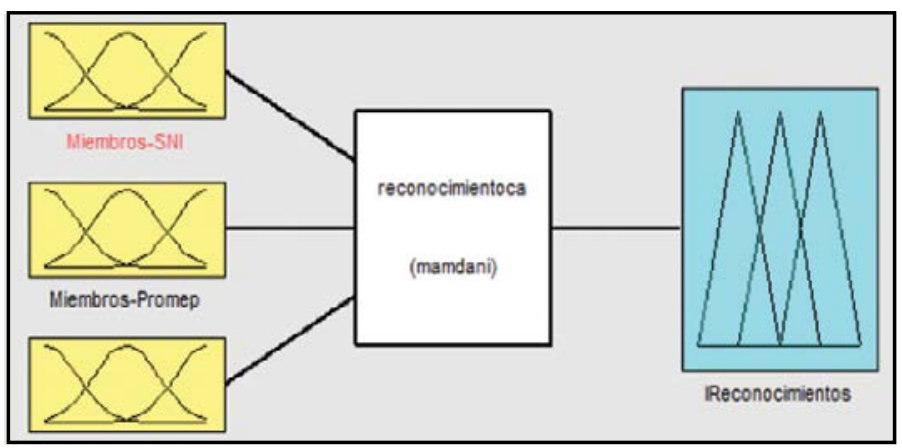

Figura 4. Sistema de inferencia difuso para el eje de Títulos Académicos. Elaboración propia.

A partir de la conformación de los cinco SID se generaron los IDPca, mediante el procedimiento de fuzzificación- desfuzzyficación se definió la salida. Para esta aplicación, el potencial de los CA se 
valoró en cinco conjuntos de tipo triangular con las etiquetas "muy mala", "mala", "regular", "buena" y "muy buena", tal como se ilustra en la Figura 5.

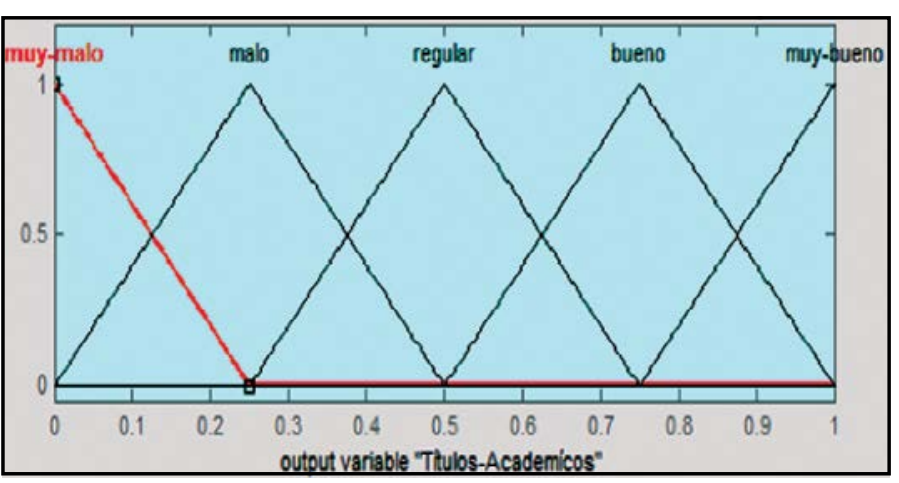

Figura 5. Variable de salida de SID. Elaboración propia.

Es pertinente hacer mención de los beneficios que supone la construcción de este tipo de conjuntos y en general del abordaje desde la teoría de la lógica difusa, posiblemente lo más significativo es su capacidad para transformar las variables que tradicionalmente se codifican dicotómicamente o por estratos, en un continuo que permite identificar diferentes matices en el análisis y por tal en los resultados obtenidos.

\section{RESULTADOS}

El análisis y cálculo del IDPca versó en el sentido de hacer el comparativo entre los cuerpos académicos que colaboran en red con algunos miembros de otros cuerpos académicos y los que no establecen redes de colaboración, lo anterior bajo cuatro principales formas de red; redes de colaboración Institucionales, redes de colaboración estatales, redes de colaboración nacionales y las redes de colaboración internacionales.

La Tabla 2 muestra el IDPca para cada una de las 59 universidades involucradas diferenciado por el tipo de redes que establecen, donde el valor del potencial más elevado es el que se ubica en la universidad 14.

\section{DISCUSIÓN}

En la Figura 6 se muestra el porcentaje de cuerpos académicos que recaen bajo una escala de clasificación con base en el potencial obtenido, a partir del rango total se realiza una subclasificación equidistante considerando tres niveles de potencial: bajo potencial, potencial medio y alto potencial. Se percibe en el primer momento una concentración en la región central del país, donde los cuerpos académicos alcanzan mayor potencial; asimismo, es evidente que en la parte sur del país se encuentran los CA con menor potencial.

El cálculo del IDPca mediante el uso de SID permite también generar índices promedio multidimensionales, la Tabla 3 muestra el valor del $\rightarrow$ IDPca por tipo de red que establece cada CA si es que hacen redes.

Tabla 2. Potencial de los cuerpos académicos

\begin{tabular}{|c|c|c|c|c|c|c|c|c|c|c|c|}
\hline Universidad & IDPca & Universidad & IDPca & Universidad & IDPca & Universidad & IDPca & Universidad & IDPca & Universidad & IDPca \\
\hline 1 & 0.3116 & 11 & 0.4587 & 21 & 0.3463 & 31 & 0.439 & 41 & 0.4416 & 51 & 0.3124 \\
\hline 2 & 0 & 12 & 0.3864 & 22 & 0.3294 & 32 & 0.4052 & 42 & 0.4205 & 52 & 0 \\
\hline 3 & 0.2883 & 13 & 0.4661 & 23 & 0.4097 & 33 & 0.3267 & 43 & 0.3511 & 53 & 0.3364 \\
\hline 4 & 0 & 14 & 0.4839 & 24 & 0.4188 & 34 & 0.2957 & 44 & 0.4342 & 54 & 0.4418 \\
\hline 5 & 0.3742 & 15 & 0.4539 & 25 & 0.4166 & 35 & 0 & 45 & 0 & 55 & 0.3958 \\
\hline 6 & 0 & 16 & 0.3867 & 26 & 0.3259 & 36 & 0.3281 & 46 & 0.1728 & 56 & 0.3035 \\
\hline 7 & 0.466 & 17 & 0.41 & 27 & 0.3588 & 37 & 0.3716 & 47 & 0.3638 & 57 & 0 \\
\hline 8 & 0.2616 & 18 & 0.4266 & 28 & 0.3659 & 38 & 0.3659 & 48 & 0 & 58 & 0.311 \\
\hline 9 & 0.4218 & 19 & 0.3989 & 29 & 0.4795 & 39 & 0.3542 & 49 & 0.2764 & 59 & 0.386 \\
\hline 10 & 0.3557 & 20 & 0.4061 & 30 & 0.3608 & 40 & 0.2378 & 50 & 0 & & \\
\hline
\end{tabular}

Elaboración propia. 


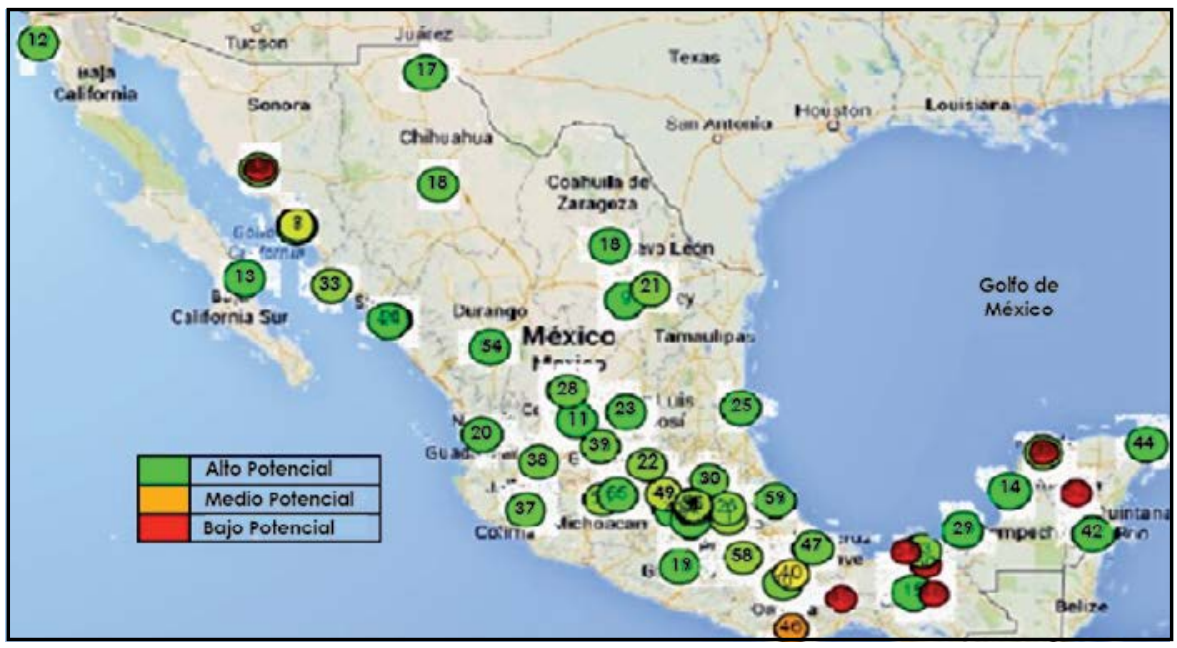

Figura 6. Grado de potencial de los CA. Elaboración propia.

Tabla 3. $\rightarrow I D P c a$ por tipo de red conformada por los CA

\begin{tabular}{|c|c|c|c|c|}
\hline & $\begin{array}{c}\text { Redes } \\
\text { institucionales }\end{array}$ & $\begin{array}{c}\text { Redes } \\
\text { estatales }\end{array}$ & $\begin{array}{c}\text { Redes } \\
\text { nacionales }\end{array}$ & $\begin{array}{c}\text { Redes } \\
\text { internacionales }\end{array}$ \\
\hline $\begin{array}{c}\text { Sí hacen } \\
\text { redes }\end{array}$ & 0.4032 & 0.4132 & 0.4076 & 0.4259 \\
\hline $\begin{array}{c}\text { No hacen } \\
\text { redes }\end{array}$ & 0.3327 & 0.3568 & 0.3332 & 0.3477 \\
\hline
\end{tabular}

Elaboración propia.

Resalta el hecho de que para los cuatro casos de tipos de redes el $\rightarrow$ IDPca es mayor en los CA que sí establecen una red de colaboración. Lo anterior faculta pensar la existencia del beneficio de la conformación de redes de colaboración. Igualmente se calculó el $\rightarrow$ IDPca por grado de consolidación de los CA, en donde es evidente el mayor potencial en los CA consolidados y que sí establecen redes de colaboración, por su parte el menor potencial recae en los CA en formación (Tabla 4). En este sentido se corrobora el beneficio de un nivel consolidado de trabajo al interior del CA, así como la conformación de redes de alguno de los cuatro tipos analizados.

El análisis permitió calcular el potencial promedio que tienen las universidades incluidas por área del conocimiento, para lo cual resaltan con mayor potencial los CA que realizan actividades
Tabla 4. $\rightarrow$ IDPca por grado de consolidación del CA

\begin{tabular}{|l|c|c|}
\hline $\begin{array}{c}\text { Grado de } \\
\text { Consolidación del } \\
\text { CA }\end{array}$ & Sí hacen redes & No hacen redes \\
\hline 1.- En formación & 0.4403 & 0.3066 \\
\hline 2.- En consolidación & 0.445 & 0.3098 \\
\hline 3.- Consolidado & 0.4573 & 0.3272 \\
\hline Elaboración propia.
\end{tabular}

de investigación y gestión en el área agropecuaria particularmente y que al igual que todas las áreas establecen redes de colaboración (Tabla 5). Es evidente otra vez la confirmación del mayor potencial en los CA que establecen algún tipo de red de colaboración. 
Tabla 5. Potencial por área del conocimiento y grado de consolidación de los CA

\begin{tabular}{|c|c|c|c|}
\hline Grado de consolidación del CA & Área del conocimiento & Sí hacen redes & No hacen redes \\
\hline \multirow{6}{*}{1} & Agropecuarias & 0.5663 & 0.2911 \\
\hline & Sociales y Administrativas & 0.3817 & 0.3055 \\
\hline & Salud & 0.5063 & 0.2979 \\
\hline & Ingeniería y Tecnología & 0.4692 & 0.2768 \\
\hline & Naturales y Exactas & 0.4121 & 0.3196 \\
\hline & Educación, Humanidades y Artes & 0.001 & 0.3033 \\
\hline \multirow{6}{*}{2} & Agropecuarias & 0.5242 & 0.3777 \\
\hline & Sociales y Administrativas & 0.4693 & 0.3379 \\
\hline & Salud & 0.3733 & 0.2993 \\
\hline & Ingeniería y Tecnología & 0.4724 & 0.3045 \\
\hline & Naturales y Exactas & 0.4557 & 0.2837 \\
\hline & Educación, Humanidades y Artes & 0.3591 & 0.2978 \\
\hline \multirow{6}{*}{3} & Agropecuarias & 0.5154 & 0.3841 \\
\hline & Sociales y Administrativas & 0.4806 & 0.3881 \\
\hline & Salud & 0.5228 & 0.2971 \\
\hline & Ingeniería y Tecnología & 0.4429 & 0.3592 \\
\hline & Naturales y Exactas & 0.5016 & 0.2513 \\
\hline & Educación, Humanidades y Artes & 0.4072 & 0.3293 \\
\hline
\end{tabular}

Elaboración propia

Tabla 6. Universidades contempladas para el estudio

\begin{tabular}{|c|c|c|c|c|}
\hline \multicolumn{5}{|c|}{ Universidades } \\
\hline $\begin{array}{l}\text { 1.-Benemérita Universidad } \\
\text { Autónoma de Puebla }\end{array}$ & $\begin{array}{l}\text { 14.-Universidad Autónoma } \\
\text { de Campeche }\end{array}$ & $\begin{array}{l}\text { 27.-Universidad Autónoma } \\
\text { de Yucatán }\end{array}$ & $\begin{array}{l}\text { 40.-Universidad } \\
\text { de la Sierra Juárez }\end{array}$ & $\begin{array}{l}\text { 53.-Universidad Juárez } \\
\text { Autónoma de Tabasco }\end{array}$ \\
\hline $\begin{array}{l}\text { 2.-Centro de Estudios } \\
\text { Superiores de Sonora }\end{array}$ & $\begin{array}{l}\text { 15.-Universidad Autónoma } \\
\text { de Chiapas }\end{array}$ & $\begin{array}{l}\text { 28.-Universidad Autónoma } \\
\text { de Zacatecas }\end{array}$ & $\begin{array}{l}\text { 41.-Universidad } \\
\text { de Occidente }\end{array}$ & $\begin{array}{l}\text { 54.-Universidad Juárez del } \\
\text { Estado de Durango }\end{array}$ \\
\hline 3.-El Colegio de México & $\begin{array}{l}\text { 16.-Universidad Autónoma } \\
\text { de Chihuahua }\end{array}$ & $\begin{array}{l}\text { 29.-Universidad Autónoma } \\
\text { del Carmen }\end{array}$ & $\begin{array}{l}\text { 42.-Universidad } \\
\text { de Quintana Roo }\end{array}$ & $\begin{array}{l}\text { 55.-Universidad Michoacano } \\
\text { de San Nicolás de Hidalgo }\end{array}$ \\
\hline 4.-El Colegio Mexiquense, A. C. & $\begin{array}{l}\text { 17.-Universidad Autónoma } \\
\text { de Ciudad Juárez }\end{array}$ & $\begin{array}{l}\text { 30.-Universidad Autónoma } \\
\text { del Estado de Hidalgo }\end{array}$ & $\begin{array}{l}\text { 43.-Universidad } \\
\text { de Sonora }\end{array}$ & $\begin{array}{l}\text { 56.-Universidad } \\
\text { Pedagógica Nacional }\end{array}$ \\
\hline $\begin{array}{l}\text { 5.-Escuela Nacional de } \\
\text { Antropología e Historia }\end{array}$ & $\begin{array}{l}\text { 18.-Universidad Autónoma } \\
\text { de Coahuila }\end{array}$ & $\begin{array}{l}\text { 31.-Universidad Autónoma } \\
\text { del Estado de México }\end{array}$ & $\begin{array}{l}\text { 44.-Universidad } \\
\text { del Caribe }\end{array}$ & $\begin{array}{l}\text { 57.-Universidad Popular } \\
\text { de la Chontalpa }\end{array}$ \\
\hline $\begin{array}{l}\text { 6.-Escuela Nacional de Conservación, } \\
\text { Restauración y Museografía }\end{array}$ & $\begin{array}{l}\text { 19.-Universidad Autónoma } \\
\text { de Guerrero }\end{array}$ & $\begin{array}{l}\text { 32.-Universidad Autónoma } \\
\text { del Estado de Morelos }\end{array}$ & $\begin{array}{l}\text { 45.-Universidad } \\
\text { del Istmo }\end{array}$ & $\begin{array}{c}\text { 58.-Universidad Tecnológica } \\
\text { de la Mixteca }\end{array}$ \\
\hline 7.-Instituto Tecnológico de Sonora & $\begin{array}{l}\text { 20.-Universidad Autónoma } \\
\text { de Nayarit }\end{array}$ & $\begin{array}{l}\text { 33.-Universidad Autónoma } \\
\text { Indígena de México }\end{array}$ & $\begin{array}{l}\text { 46.-Universidad } \\
\text { del Mar }\end{array}$ & 59.-Universidad Veracruzano \\
\hline $\begin{array}{l}\text { 8.-Instituto Tecnológico } \\
\text { Superior de Cajeme }\end{array}$ & $\begin{array}{l}\text { 21.-Universidad Autónoma } \\
\text { de Nuevo León }\end{array}$ & $\begin{array}{l}\text { 34.-Universidad Autónoma } \\
\text { Metropolitana }\end{array}$ & $\begin{array}{l}\text { 47.-Universidad } \\
\text { del Papaloapan }\end{array}$ & \\
\hline $\begin{array}{l}\text { 9.-Universidad Autónoma } \\
\text { Agraria Antonio Narro }\end{array}$ & $\begin{array}{c}\text { 22.-Universidad Autónoma } \\
\text { de Querétaro }\end{array}$ & $\begin{array}{l}\text { 35.-Universidad Autónoma } \\
\text { Yucatán }\end{array}$ & $\begin{array}{l}\text { 48.-Universidad Intercultural } \\
\text { de Chiapas }\end{array}$ & \\
\hline $\begin{array}{l}\text { 10.-Universidad Autónoma } \\
\text { Benito Juárez de Oaxaca }\end{array}$ & $\begin{array}{l}\text { 23.-Universidad Autónoma } \\
\text { de San Luis Potosí }\end{array}$ & $\begin{array}{l}\text { 36.-Universidad de Ciencias } \\
\text { y Artes de Chiapas }\end{array}$ & $\begin{array}{l}\text { 49.-Universidad Intercultural } \\
\text { del Estado de México }\end{array}$ & \\
\hline $\begin{array}{l}\text { 11.-Universidad Autónoma } \\
\text { de Aguascalientes }\end{array}$ & $\begin{array}{l}\text { 24.-Universidad Autónoma } \\
\text { de Sinaloa }\end{array}$ & 37.-Universidad de Colima & $\begin{array}{l}\text { 50.-Universidad Intercultural } \\
\text { del Estado de Tabasco }\end{array}$ & \\
\hline $\begin{array}{l}\text { 12.-Universidad Autónoma } \\
\text { de Baja California }\end{array}$ & $\begin{array}{c}\text { 25.-Universidad Autónoma } \\
\text { de Tamaulipas }\end{array}$ & $\begin{array}{l}\text { 38.-Universidad de } \\
\text { Guadalajara }\end{array}$ & $\begin{array}{l}\text { 51.-Universidad Intercultural } \\
\text { Indígena de Michoacán }\end{array}$ & \\
\hline $\begin{array}{l}\text { 13.-Universidad Autónoma } \\
\text { de Baja California Sur }\end{array}$ & $\begin{array}{c}\text { 26.-Universidad Autónoma } \\
\text { de Tlaxcala }\end{array}$ & $\begin{array}{l}\text { 39.-Universidad de } \\
\text { Guanajuato }\end{array}$ & $\begin{array}{l}\text { 52.-Universidad Intercultural } \\
\text { Maya de Quintana Roo }\end{array}$ & \\
\hline
\end{tabular}


CONCLUSIONES

La cuantificación del potencial de los cuerpos académicos permite evidenciar la relevancia que tiene la construcción de redes de cooperación, en los casos analizados es notorio el incremento que se genera en la capacidad de acción de los CA que tienen relaciones con otros CA. A pesar de que en la esfera institucional es en donde se evidencian mayores diferencias, en los tres tipos de redes se visualizan mejores condiciones para los CA que sí establecen redes de cooperación.

El análisis presentado en este trabajo comprueba los planteamientos de la teoría de construcción de redes, que establece mejores niveles -en este caso- de producción y generación del conocimiento; es decir, la cooperación al interior de las universidades como al exterior entre CA, podrá propiciar mejoras en la generación de conocimiento científico.
El estudio de fenómenos sociales, y en específico el análisis y cuantificación del potencial de CA a partir de su productividad y la conformación de redes, ha encontrado una elemental distinción, de ser multidimensional y totalmente dinámico, pocas y cada vez menos veces explicado de forma estática y unidimensional. En este sentido el uso de la teoría de la lógica ha permitido en primer momento un análisis de forma multidimensional, así como mayor precisión en el cálculo del IDPca. Sin embargo, es pertinente resaltar, que el actual trabajo podrá alcanzar una mayor profundidad en el análisis, en tanto se puedan incluir en la metodología de cuantificación más ejes de análisis que puedan ser representativos del total de las actividades que realizan los Cuerpos Académicos en México y que es precisamente la continuidad que se seguirá en esta línea de investigación y queda como propuesta para futuros trabajos que complementen el presente y permitan abonar al conocimiento de la producción científica y el análisis de cuerpos académicos.

LITERATURA CITADA

- BANCO INTERNACIONAL DE RECONSTRUCCIÓN Y FOMENTO/BANCO MUNDIAL. Construyendo Sociedades del Conocimiento: Nuevos Desafíos para la Educación Terciaria. Washington, DC, EE. UU.: Autor, 2003.

- CARAYOL, N. y ROUX, P. Knowledge flows and the geography of networks: A strategic model of small world formation. Journal of Economic Behavior \& Organization, 71 (2): 414-427, 2009.

- CARAYOL, N. et al. Inefficiencies in a model of spatial networks formation with positive externalities. Journal of Economic Behavior \& Organization, 67(2): 495-511, 2008.

- FLORES, L. y CAMARENA, M. Evaluación de programas públicos en el marco de la realidad social, metodología basada en la lógica difusa como instrumento para el análisis de fenómenos sociales. Revista Latinoamericana de Metodología de la Investigación Social-ReLMIS, 3(5): 8-23, 2013.

- FlORES PAYÁN, L. y GARCÍA BATíz, M. L. Evaluación de programas públicos mediante lógica difusa: el caso del Programa Hábitat. Política y Cultura, otoño 2013(40): 231-255, 2013.

- JACKSON, M. O. Social and economic networks. Princeton: Princeton University Press, 2008.
- JACKSON, M. O. The economics of social networks. En R. Blundell, W. Newey \& T. Persson (Eds.), Proceedings of the $9^{\text {th }}$ World Congress of the Econometric Society (1-56). Cambridge, UK: Cambridge University Press, 2005.

- JACKSON, M. O. y WOLINSKY, A. A strategic model of social and economic networks. Journal of Economic Theory, 71 (1): 44-74, 1996.

- KOSSINETS, G. y WATTS, D. Origins of homophily in an evolving social network. American Journal of Sociology, 115(2): 405450, 2009.

- LÓPEZ, A. et al. Descomposición de la pobreza multidimensional mediante el enfoque de conjuntos difusos: una aplicación para el México rural. Documento de trabajo del CIDE-División de Economía, 464, 2009.

- SALAS, I. y MURILLO, F. Las microinteracciones como el origen de las redes de cooperación. Revista Latinoamericana de Metodología de la Investigación Social, 4(7): 57-77, 2014.

- ZADEH, L. A. Fuzzy sets. Information and Control, 8(3): 338-353, 1965. 